\title{
You Can Observe a Lot by Watching: How Artistic Images have Led to Anatomical Exactitude
}

\author{
Emmanuelle Hirt', Skylar LaManna', Francisco Abellan², Gregorio Ramirez², David Kilroy1,*
}

\begin{abstract}
Since the publication (c. 1316) of the first anatomy textbook and dissection guide, Anothomia mundini by Mondino de Luzzi, the importance of illustrations has been recognised as essential to learning about body structure. The Anathomia was the main source of anatomy information for over two centuries until Vesalius produced a comprehensive atlas of human anatomy, published in 1543 as De humani corporis fabrica. This text contained over 200 illustrations and placed considerable emphasis on dissection and observation as the best method of studying anatomy, a departure from the long-standing tradition of following ancient texts which lacked illustrations. This empirical method was expanded by several artists, notably Leonardo da Vinci and George Stubbs, whose works laid the foundation for accuracy in anatomical illustrations. The current tendency among students to photograph their dissections has largely replaced drawing specimens. Some contemporary antidotes to this tendency are presented along with the associated educational benefits.
\end{abstract}

Key words: 'Dark Ages' myth, Vesalius, Carlo Ruini, Directly-observed art, Visual learners.

\section{Development of Anatomy}

Before the establishment in the late middle ages of universities, anatomical education was based on a thorough study of books and by animal dissection. For several centuries human dissection was thought unnecessary due to the comprehensive works of such ancient scholars as Hippocrates, Aristotle and Galen. In addition, the prevailing beliefs and laws of Greek and Roman societies which forbade dissection of the human corpse persisted until the $12^{\text {th }}$ century CE, a fact often ignored by many publications, ${ }^{1,2}$ which prefer to attribute this position to the now thoroughly discredited myth of the 'dark' ages. With the foundation of universities, especially in Italy and the expansion of medical education, human dissection became an integral part of the anatomy curriculum. Teaching initially consisted of a professor standing at an elevated lectern reading to an attentive student audience (Figure 1). Occasionally, instructions from a classical text were dispensed to an assistant who dissected the cadaver. An anatomy professor in Bologna, Mondino de Luzzi (c1276-1326) is credited with having performed the first human dissection and his textbook Anothomia contained numerous illustrations and a dissection guide, which made it the definitive work of anatomy for the next two centuries.

Prior to the publication of Anathomia, students were taught from the classical works of Galen ( $2^{\text {nd }}$ century $\mathrm{CE}$ ) which contained numerous errors, probably as a result of minimal human dissection and a reliance on animal models for information. While de Luzzi's work was a major milestone in the development of anatomy, it contained many factual errors (a sevenchambered uterus, whereas Galen had two chambers in his description of the uterus). Any deviation from the historical texts was deemed to be due to morphological transmutation. ${ }^{1}$ It was not until the $16^{\text {th }}$ century CE and the publication of Vesalius' comprehensive text De humani corporis fabrica that the emphasis on observation and careful study of anatomical specimens became well established. Since the advent of the printing press in the mid-fifteenth century, numerous texts had been published and this aided the expansion in knowledge in many domains, including the discipline of anatomy. However, Vesalius' work became the standard anatomy reference book due in part to its many excellent and accurate illustrations (Figure 2). Vesalius relied on direct observation of dissected specimens to produce his Fabrica and this approach was the initial step in promoting an evidence-based subject in contrast to the previous reliance on the authority of the classical anatomists. Figure 2, which shows the human skull on top of the canine skull, appears twice in Book I of the Fabrica and its presence was to contradict Galen's assertion that the premaxillary bone and suture of the dog were present in humans, an argument that was settled in favour of Vesalius only after several centuries of academic wrangling. ${ }^{3}$ Some errors in

\section{Emmanuelle Hirt ${ }^{1}$, Skylar LaManna ${ }^{1}$, Francisco Abellan², Gregorio Ramirez ${ }^{2}$, David Kilroy ${ }^{1, *}$ 'School of Veterinary Medicine, University College Dublin, Belfield, Dublin, IRELAND. \\ ${ }^{2}$ Department of Veterinary Anatomy, Faculty of Veterinary Medicine, University of Murcia, SPAIN.}

\section{Correspondence}

Dr. David Kilroy, MRCVS

School of Veterinary Medicine, University College Dublin, Belfield, Dublin-04, IRELAND.

Phone no: 35317166235 ;

E-mail: david.kilroy@ucd.ie

History

- Submission Date: 11-03-2020

- Review completed: 12-04-2020

- Accepted Date: 27-04-2020.

DOI : 10.5530/bems.6.2.5

Article Available online

http://www.bemsreports.org/v6/i2

Copyright

(c) 2020 Phcog.Net. This is an openaccess article distributed under the terms of the Creative Commons Attribution 4.0 International license. 
anatomy did appear in the Fabrica, probably as a result of using animal specimens instead of human cadavers. For example, the retractor bulbi muscle, a well-developed extrinsic eye muscle in dogs, cattle and other domestic animals, is shown in the dissected human eye from which it is absent. The illustration of the hyoid bone is taken from a canine model, as is the drawing of the tongue and associated musculature.

\section{Innovations in Art}

Alongside the flourishing educational world, artistic advances were equally significant in advancing anatomy and one of the most prominent artists in this process was Leonardo da Vinci (1452-1519) whose many achievements include numerous careful, extremely accurate drawings of the human body (Figure 3). These illustrations were often based on the study of dissected human cadavers which were not preserved and must have been dissected with great care and skill. ${ }^{4}$ Over the next few centuries, Leonardo's methods of direct observation became established among the artistic community. The only significant text on equine anatomy produced during this period was Carlo Ruini's Dell'anotomia, e dell'infermita del cavallo, which was published posthumously in 1598 and contained superb woodcut illustrations by an unknown artist, described in the text as a 'Student of Titian'. ${ }^{5}$ The considerable attention to detail and the anatomical accuracy of the woodcuts can clearly be seen (Figure 4). This remained the defining work on equine anatomy until the arrival of the English artist George Stubbs (1724-1806) who studied human anatomy in York before devoting his time to the anatomy of the horse. Again, meticulous dissection and careful attention to detail was combined with Stubbs' artistic genius to produce a comprehensive set of etchings titled Anatomy of the Horse, published in 1776. As shown in Figure 5, Stubbs illustrated the anatomy of the horse in a vivid manner and his work has undergone a deserved revival in recent decades. ${ }^{5}$

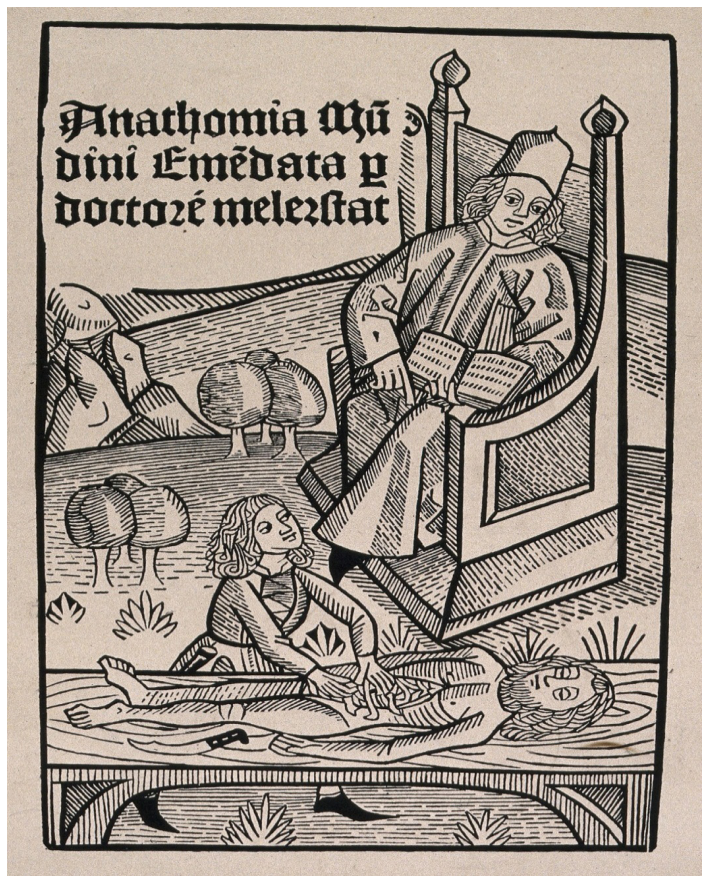

Figure 1: A man seated in a chair in a landscape, holding an open book, directing a dissection which is taking place in the foreground. Line block after a woodcut, c. 1493. Credit: Wellcome Collection.

\section{Current Approaches to Anatomy}

Dissection is still the cornerstone of anatomy teaching and veterinary students typically use the dog as the model for their studies. In recent years, the inclination of students to sketch or draw their anatomical work has been replaced by phone photos or videos of the dissection which is then used for revision prior to assessments. However, drawing images is an active process and has made significant contributions to many scientific disciplines, including anatomy. ${ }^{6}$ The superior retention of knowledge and a deeper understanding of structure and function is helped by the activity of drawing. The required creativity is a whole-brain process and artists and scientists use the same parts of their brains to do complex, creative tasks. ${ }^{7}$ Ensuring that students understand the value of drawing to their learning can help motivate them to undertake this task. Using the work of students who are skilled artists can assist with this (Figures 6 to 10).

For those who are visual learners, drawing sketches can reinforce both the understanding and recall of important anatomical features. In the words of artist Emmanuelle Hirt, 'The initial goal of these drawings was to offer a descriptive, life-like picture of a certain feature of an animal currently being studied and to use this drawing for revision. The actual process and act of drawing from specimens allows a slow perusal of every part of the animal and enables the piecing together of a larger structure from its components. Once the general structure is sketched, attempts are made to replicate colors and textures of the original specimen to enhance learning and ability to recognize these structures on either a live animal or in dissection. Enhanced shading can show depth of structures and can further help to replicate the look of a live specimen. The hard copy of a drawing may seem cumbersome, but is especially nice when reviewing to avoid looking at a computer screen. Students can also make these drawings of varying sizes to help emphasize certain areas or to even make them life sized. Labelling an image allows the

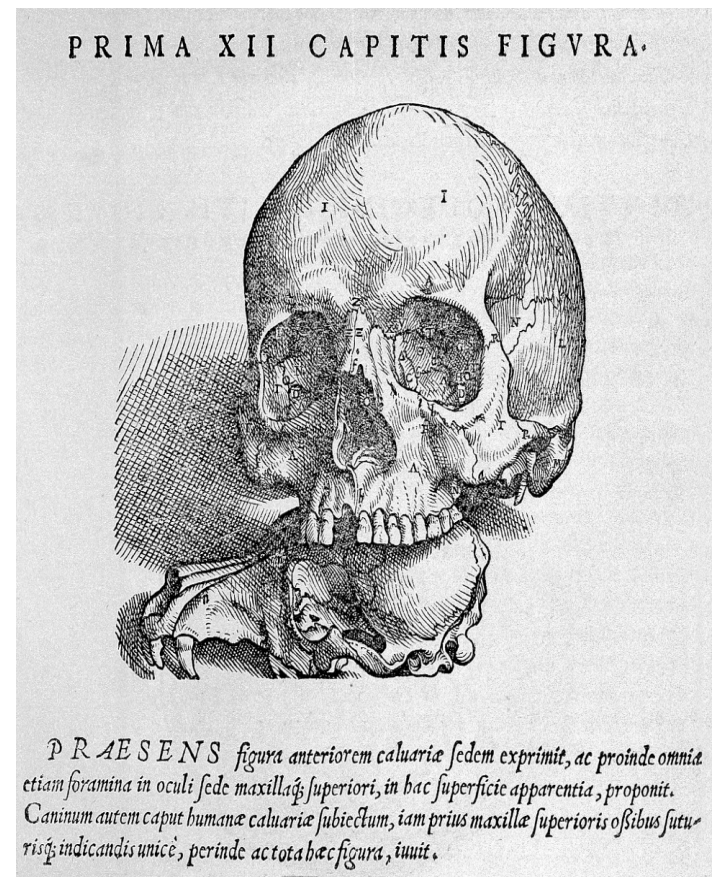

Figure 2: A. Vesalius, skull, with one line of text. From De humani corporis fabrica Credit: Wellcome Collection. 
student to have an immediate understanding of the muscle, bone, etc. that they are studying. Artistic media is an important study tool the students can utilize to further their comprehension of animal anatomy and overall body function. 'As seen in Figures 7 and 9 (produced by Skylar LaManna), the use of various applications on a tablet with an electronic pencil provides the same freedom of expression as with the

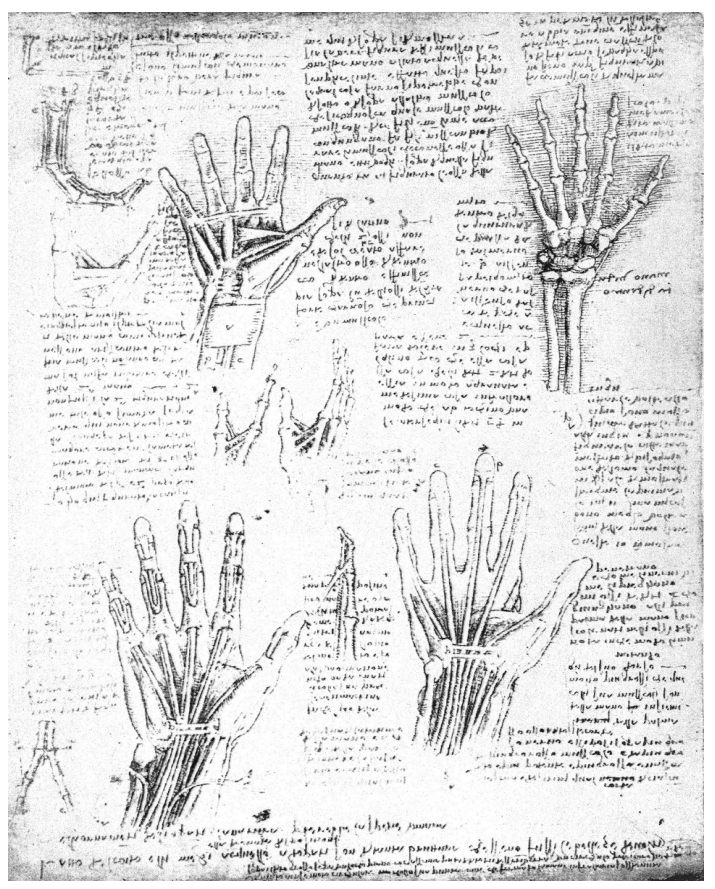

Figure 3: Figure 41"Leonardo da Vinci the anatomist" from McMurrich. Credit: Wellcome Collection.

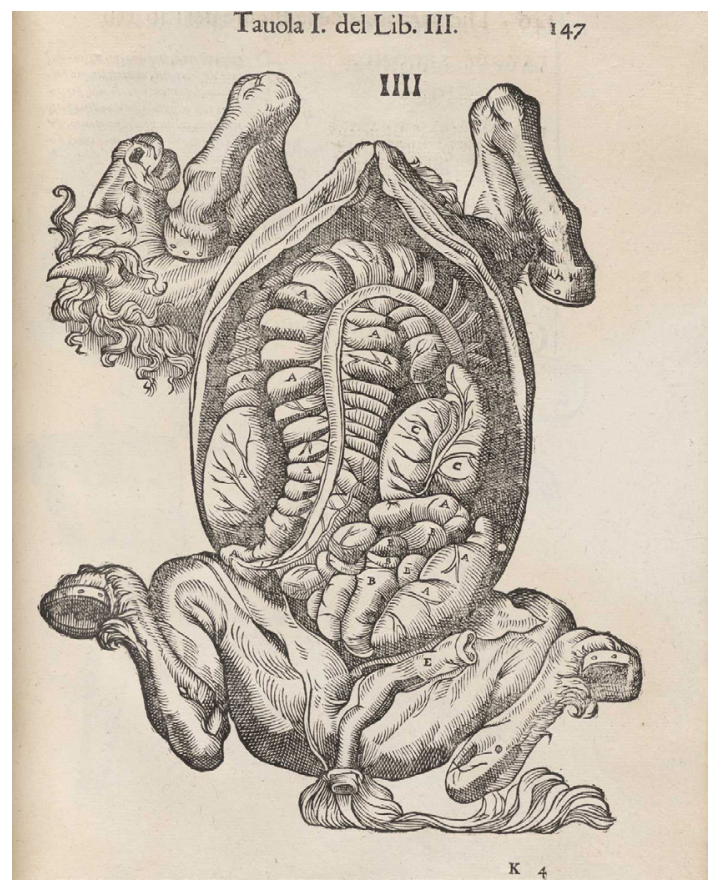

Figure 4: Image of the equine large intestine: woodcut from Ruini's Dell'anotomia, e dell'infermita del cavallo page 147. Credit:National Library of Medicine. traditional paper-based approach. The anatomical structures are drawn using a mixture of basic sketching techniques such as cross hatching or blending. Through the application of distinguishing colours to the various anatomical features, different structures can easily be identified, which aids the visual learner. This type of two-dimensional medium has such a wide range of capabilities for recreation of three-dimensional anatomical structures in different ways that help to better understand their functions and relationships. This is an extremely helpful technique to gain a deeper understanding of the essential materials expected of veterinary students.

In addition, drawing of specimens give a true representation of what is seen and can show the biological variation that exists among different species or within these groups, an important factor which is often overlooked when studying from textbooks. Figure 8 is an illustration of a canine forelimb with the muscles labelled and additional student comments added as revision aids. This image is an accurate representation of the greyhound forelimb and neatly conveys its extensive muscular development, which is a feature of this breed and is generally not replicated in other dogs. This type of image, especially when student generated, can emphasise to the class the extent of biological variation that occurs across different animal species and breeds.

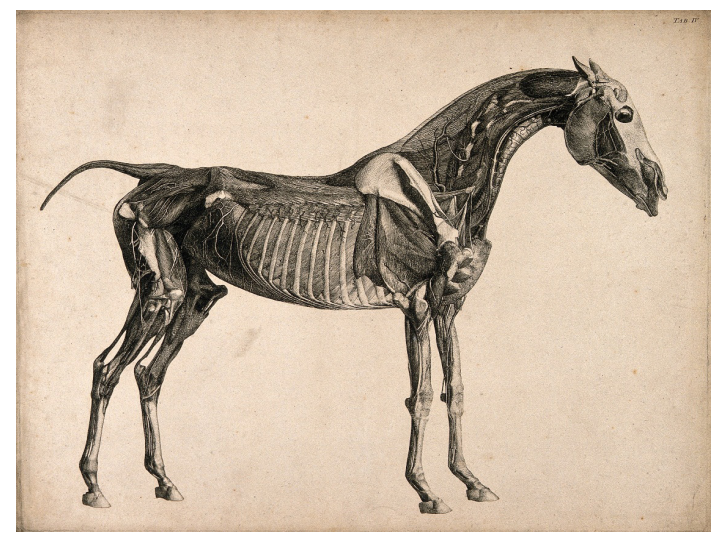

Figure 5: Muscles, bones and blood-vessels of a horse: side view. Engraving with etching by G. Stubbs, 1766. Credit: Wellcome Collection.

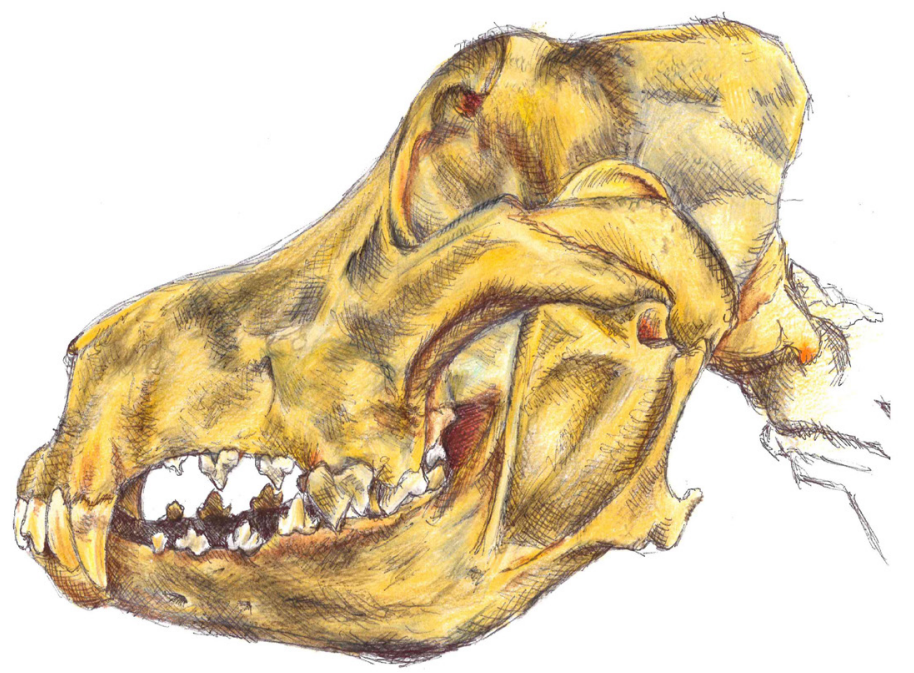

Figure 6: Sketch of a canine skull, lateral view. Credit: Emma Hirt (artist). 


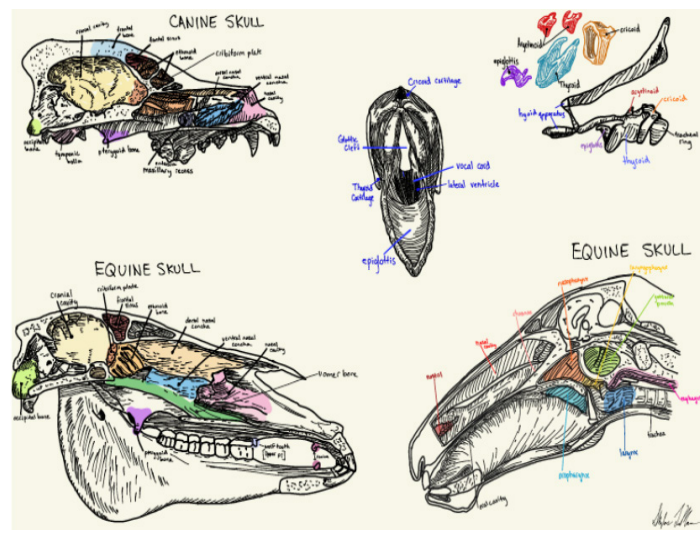

Figure 7: Canine musculature of proximal left limb (lateral and medial aspects) and cranial view. Credit: Skylar LaManna (artist).

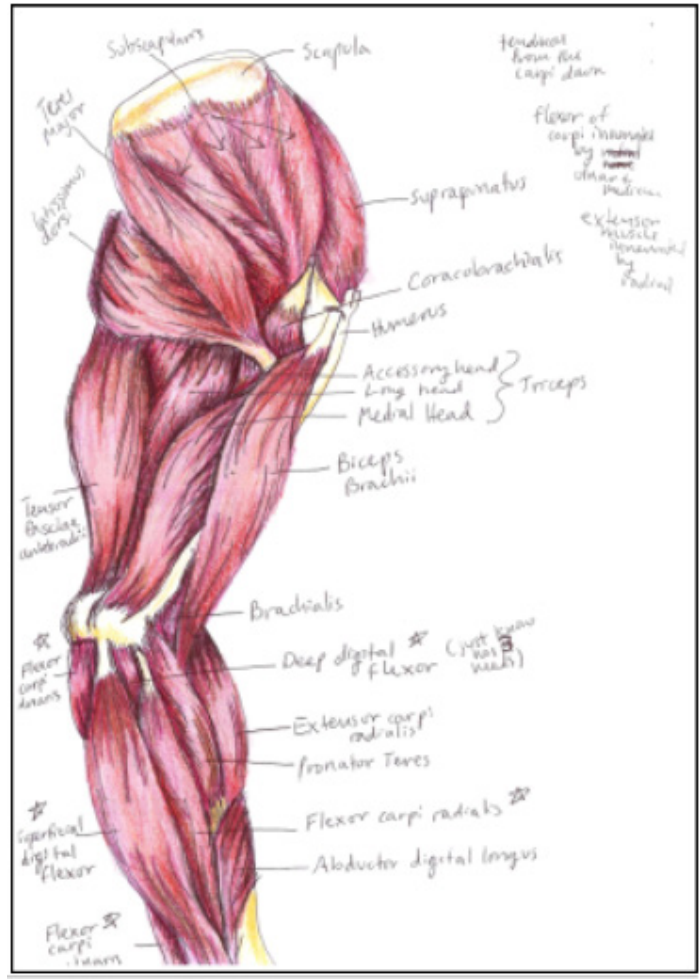

Figure 8: Coloured illustration of a canine forelimb (greyhound), medial view. Credit: Emmanuelle Hirt (artist).

Blending traditional drawing with modern technology can merge the artistic skills of the student with computer technology, as shown in Figure 9, which combines three images to give a 3-dimensional view of the canine forelimb. Annotations have been added by the student artist and the digital image can be studied on a computer or mobile device, thereby expanding its usefulness for learning and revision. A similar approach can be seen in Figure 10, which shows two stags butting heads. In addition, the bones of the limbs have been made visible and the range of movement of the limb joints are also illustrated with anatomical accuracy, which can serve to emphasise the functional anatomy of locomotion.

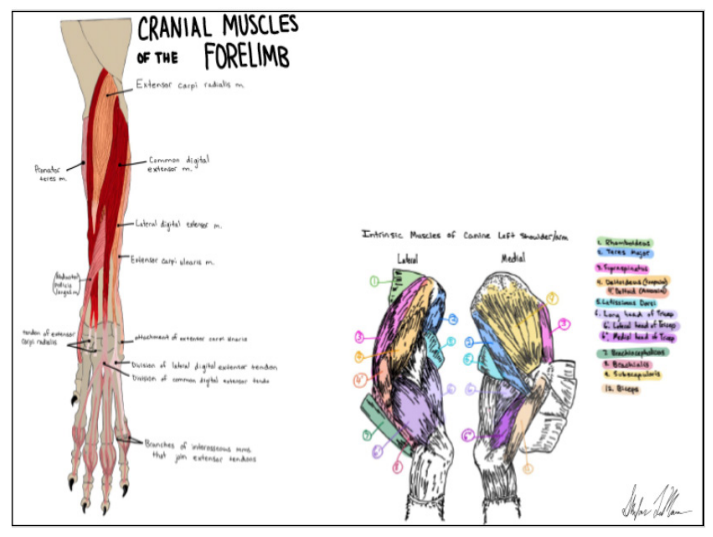

Figure 9: Canine musculature of proximal left limb (lateral and medial aspects) and cranial view. Credit: Skylar LaManna (artist).

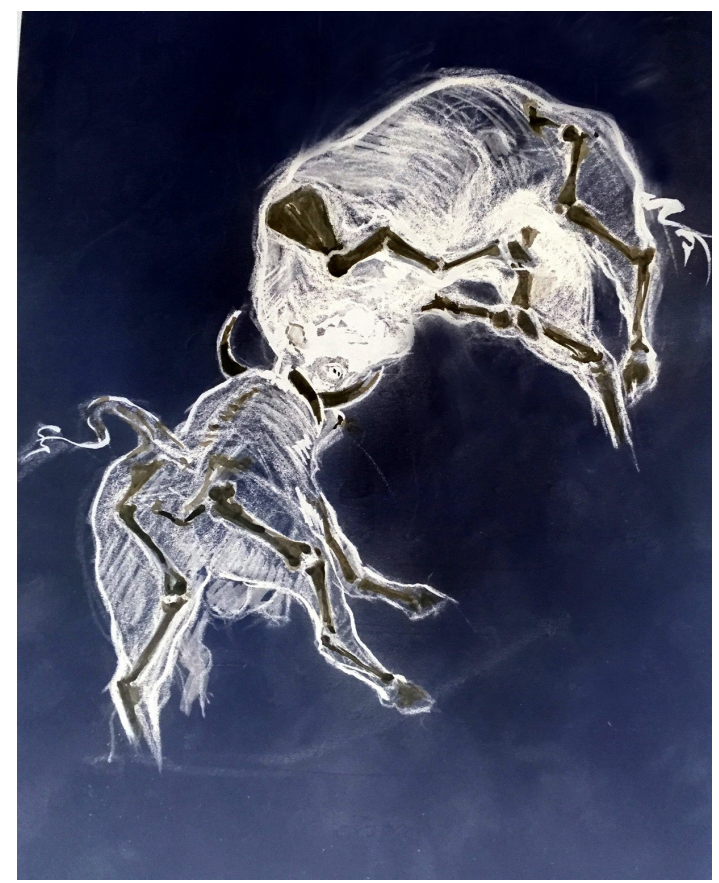

Figure 10: Two stags clashing, with bones of forelimbs and hindlimbs rendered visible. Credit: Dr Francisco Abellan (artist).

\section{SUMMARY AND CONCLUSIONS}

Since its move to an empirical approach in the $13^{\text {th }}$ century CE, anatomy and art have collaborated in the development and progress of both subjects, with some of the most illustrious artists (Leonardo and Michelangelo) exhibiting a commendable commitment to anatomical accuracy and detail. The current teaching approach to anatomy uses dissection as its primary means of learning. Using skilled artists, especially students, can stimulate discussion, lead to a greater appreciation of biological variation and encourage direct observation as a means of deeper learning. A combination of research and observations from dissection, diagrams in textbooks and previous experiences, are translated into the images which provide a more tangible grasp of concept. 


\section{ACKNOWLEDGEMENT}

Many thanks to the three artists who contributed their images to this paper: Emmanuelle Hirt and Skylar LaManna are currently veterinary students at University College Dublin, Ireland. Dr Abellan is a veterinarian and a faculty member of the Universidad de Murcia, Spain.

\section{CONFLICT OF INTEREST}

None.

\section{ABBREVIATIONS}

c: circa; CE: Common Era.

\section{REFERENCES}

1. Crivellato E, Ribatti D. Mondino de' Liuzzi and his Anothomia: A milestone in the development of modern anatomy. Clin Anat. 2006;19(7):581-7.

2. Ghosh SK. Evolution of illustrations in anatomy: A study from the classical period in Europe to modern times. Anat Sci Educ. 2015;8(2):175-88.

3. Saunders JB. De CM, O'Malley CD. The Illustrations from the Works of Andreas Vesalius of Brussels. Dover Fine Art, History of Art. 1973.

4. Jones R. Leonardo da Vinci: Anatomist. British Journal of General Practice. 2012;62:319.

5. Taylor B. Stubbs. London: Phaidon Press. 1971.

6. Merkle BG. Drawn to Science. Nature. ;562:S8-9.

7. Andreasen NC, Ramchandran K. Creativity in art and science: Are there two cultures?. Dialogues Clin Neurosci. 2012;14(1):49-54.

Cite this article : Hirt E, LaManna S, Abellan F, Ramirez G, Kilroy D. You can Observe a lot by Watching: How Artistic Images have Led to Anatomical Exactitude. BEMS Reports. 2020;6(2):14-8. 